\title{
INTERMEDIATE INEQUALITY AND WELFARE: THE CASE OF SPAIN, 1980-81 TO 1990-91
}

\author{
By CORAl Del Río
}

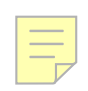

Universidade de Vigo

AND

JAVIER Ruiz-CASTILLO

Universidad Carlos III de Madrid

The 1990-91 household expenditures distribution in Spain dominates, in the relative ("rightist") Lorenz sense, the 1980-81 distribution, but the latter dominates the former in the absolute ("leftist") Lorenz sense. This situation constitutes a textbook case for intermediate or "centrist" notions of inequality and social welfare. This paper presents the first empirical application of this sort, using the intermediate inequality concept introduced in Del Río and Ruiz-Castillo (2000). The data reveal that there is a decrease in household expenditures inequality for a relatively small set of centrist attitudes.

\section{INTRODUCTION}

This paper studies the evolution of household expenditures inequality and social welfare in Spain during the 1980s. This is an interesting period of highly unusual political characteristics, in which the Socialist party occupied power through democratic means for the first time in 40 years. ${ }^{1}$

Using standard methods developed by Shorrocks (1983) and Moyes (1987), Del Río and Ruiz-Castillo (1996) found that the 1990-91 household expenditures distribution in Spain had a greater mean, less relative inequality but more absolute inequality than the 1980-81 comparable distribution. ${ }^{2}$ However, the following empirical question cannot be answered with the present tools: is the 1990-91 expenditures distribution in Spain "barely better" than the 1980-81 distribution from the relative point of view, and consequently "far away" from the absolute one; or is it "so much better" from the relative perspective that it is "nearly equivalent" to it from the absolute point of view? In other words, present methods only address yes-or-no questions relative to the two polar cases, but in situations

Note: This work is part of the first author's $\mathrm{PhD}$ dissertation, and has been carried out under the auspices of the ECC TMR Contract \#ERBFMRXCT980248. Financial help from Project PB960118 of the Spanish DGES is gratefully acknowledged.

${ }^{1}$ Recall that Spain achieved a democratic regime during the mid 1970 s, and became a full member of the European Community in 1986. Ever since 1978, Spain has been involved in a complex process of economic modernization and liberalization, while striving at the same time to catch up for lost time in the setting up of a Welfare State comparable to that existing in other Western societies. For the development of the Spanish economy over the last four decades, see Martin (1999).

${ }^{2}$ Except for Portugal, which has gone through similar political and economic reforms since the mid 1970s, this is a different trend from most OECD countries. For Portugal, see Gouveia and Tavares (1995) and Rodrigues (1993); for the international experience see, for instance, Atkinson, Rainwater, and Smeeding (1995) and Gottschalk and Smeeding (1997); for Spain, see Del Río and Ruiz-Castillo (1996, 2001); and for a comparison between Portugal and Spain, see Jimeno et al. (2000). 
like the Spanish one during the 1980s they remain silent on whether the decrease in relative inequality (or the increase in absolute inequality) is "large" or "small."

In order to provide an answer to this question, it is reasonable to use "centrist" or intermediate notions of inequality between the "rightist" (relative) and the "leftist" (absolute) cases in Kolm (1976a, 1976b) terminology. This paper presents the first empirical application of an intermediate inequality concept and the associated class of intermediate social welfare functions. The intermediate inequality notion used is the one introduced in Del Río and Ruiz-Castillo (2000) - DRRC, for short - which depends on an initial income or expenditures distribution $\mathbf{x}$ and a parameter value $\pi$ in the unit interval. Two income distributions $\mathbf{x}$ and $\mathbf{y}$ are said to have the same $(\mathbf{x}, \pi)$-inequality if the total income difference between them is allocated among the individuals as follows: $\pi 100$ percent preserving income shares in $\mathbf{x}$, and $(1-\pi) 100$ percent in equal absolute amounts.

The problem, of course, is that there are no a priori reasons to determine which centrist attitudes, or which range of $\pi$ values, should be adopted to compare any given pair of income distributions. This paper develops an operational procedure to allow the data to reveal this to us: we estimate the range of $\pi$ values, $\left(\pi_{1}, \pi_{2}\right)$ in the interval $[0,1]$, for which the 199091 and the 198081 distributions are equivalent in terms of inequality. In this way, it is learned for what type of intermediate attitudes there has been a reduction in inequality-for people with "center-right" attitudes in the interval $\left[\pi_{2}, 1\right]$ - or an increase in inequality-for people with "center-left" attitudes in the interval $\left[0, \pi_{1}\right]$. Then, taking into account the mean income change during this period, one can unambiguously conclude whether the 1990-91 income distribution is superior, inferior or noncomparable to the 1980-81 distribution for all centrist social evaluation functions in an appropriately defined class.

To apply this methodology in practice, it must be extended to the heterogeneous case in which individuals come grouped in households with different non-income needs. In this paper, household size is taken as the only household characteristic defining ethically relevant non-income needs. To pool all households in a common distribution, in the relative case Buhmann et al. (1988) and Coulter, Cowell, and Jenkins (1992a, 1992b) suggest a parametric model of equivalence scales which allows for different views about the importance of economies of scale in consumption within the household. Based on the ideas presented in Ruiz-Castillo (1998) for the absolute case, the model is extended to the intermediate case and the connection between the parametrization of economies of scale in the three cases is established.

The data used are the two latest Encuestas de Presupuestos Familiares (EPFs for short), conducted by the INE (Instituto Nacional de Estadistica) from April 1980 to March 1981 and from April 1990 to March 1991. These are comparable household budget surveys, used primarily for estimating the official weights of the Consumer Price Index. They provide the best microeconomic information on the evolution of the standard of living in Spain during this period.

For the population as a whole, the main result of the paper is that there is a decrease in household expenditures inequality for a relatively small set of centrist attitudes. For a second small group, with views slightly further to the left in the 
political spectrum, inequality differences are not statistically significant. For the rest of the population, inequality has increased. The results for the subgroups in the partition by household size, indicate that the reduction in intermediate inequality is much greater for smaller than for larger households.

The rest of the paper is divided into four sections and an Appendix. Section I justifies the use of intermediate inequality notions in situations like the Spanish one during this period. Section II presents the concept of intermediate inequality used in the paper, and describes how it can be made operational by using Lorenz comparisons, both in the homogeneous and the heterogeneous cases. Section III contains the empirical results, while section IV concludes. The Appendix contains the results for the partition by household size.

\section{Why Should We Use Intermediate Inequality Measures?}

Traditionally, welfare economics is concerned with evaluation methods which take into account an efficiency preference for larger incomes, and an equity preference for less vertical inequality. Moreover, it is desirable that empirical methods call for a minimum of value judgments. In particular, it is desirable to have unambiguous rankings according to which social welfare increases only if efficiency and distribution both improve. Let $W_{R}$ and $W_{A}$ denote the two classes of SEFs which, apart from the usual assumptions (continuity, $S$-concavity and population replication invariance - see Lambert (1993) for a discussion), have a social preference for efficiency consistent with the relative and absolute inequality notions, respectively. Shorrocks (1983) and Moyes (1987) develop operational procedures in order to investigate whether one income or expenditures distribution is better than another for all of the SEFs belonging, respectively, to each of these two classes.

Let us assume that two income distributions $\mathbf{x}$ and $\mathbf{y}$ in two different moments in time must be compared, and assume that distribution $\mathbf{y}$ has a greater mean than $\mathbf{x}$. If distribution $\mathbf{y}$ dominates $\mathbf{x}$ in the absolute Lorenz sense, then $\mathbf{y}$ is better than $\mathbf{x}$ according to all SEFs in $W_{A}$. This should be the end of the analysis, because who would deny that there has been an unambiguous increase in social welfare? Only people who believe that, to maintain inequality constant, any excess income should be distributed so as to assign greater absolute amounts to the poor than to the rich.

Suppose, however, that distribution $\mathbf{y}$ dominates distribution $\mathbf{x}$ in the relative Lorenz sense, but that $\mathbf{x}$ dominates $\mathbf{y}$ in the absolute Lorenz sense. ${ }^{3}$ This is exactly the case in Spain during the 1980s, where $\mathbf{y}$ is the 1990-91 household expenditures distribution and $\mathbf{x}$ is the $1980-81$ distribution. The situation is summarized in Table 1. Column 1 indicates that there is a general increase in mean expenditures, especially among households within the first six deciles, whose mean expenditures grow above the average. Correspondingly, expenditures' shares by deciles in 1990-91 (column 3) are larger than in 1980-81 (column 2), pointing out toward relative Lorenz dominance by the 1990-91 expenditures distribution. Finally,

${ }^{3}$ For some SEFs more sensitive to the increase in inequality than to the increase in the mean, distribution $\mathbf{y}$ may show a lower welfare level in the absolute sense, whereas other indices with the opposite characteristics may lead to the opposite conclusion. 
TABLE 1

Relative and Absolute Increases in Mean Household Expenditures

During the 1980s in Spain, and Expenditures' Shares by Deciles in 1980-81 VERSUS 1990-91

\begin{tabular}{|c|c|c|c|c|}
\hline \multirow[b]{2}{*}{ Deciles } & \multirow{2}{*}{$\begin{array}{l}\text { Relative Increase } \\
\text { in Expenditures }\end{array}$} & \multicolumn{2}{|c|}{ Expenditures' Shares } & \multirow{2}{*}{$\begin{array}{l}\text { Absolute Increase } \\
\text { in Expenditures }\end{array}$} \\
\hline & & $1980-81$ & $1990-91$ & \\
\hline 1 & 37.5 & 3.13 & 3.39 & 142,570 \\
\hline 2 & 31.7 & 4.90 & 5.09 & 188,900 \\
\hline 3 & 29.9 & 6.05 & 6.20 & 219,688 \\
\hline 4 & 29.4 & 7.08 & 7.23 & 252,857 \\
\hline 5 & 29.0 & 8.15 & 8.28 & 286,651 \\
\hline 6 & 28.4 & 9.30 & 9.42 & 321,041 \\
\hline 7 & 27.4 & 10.66 & 10.69 & 354,112 \\
\hline 8 & 26.5 & 12.39 & 12.37 & 399,001 \\
\hline 9 & 24.3 & 15.09 & 14.78 & 445,599 \\
\hline 10 & 23.0 & 23.25 & 22.55 & 649,667 \\
\hline Total & 26.8 & 100.0 & 100.0 & 326,062 \\
\hline
\end{tabular}

Note: Adjusted expenditures distribution = unadjusted expenditure distribution/

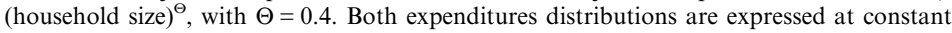
prices of the 1991 winter.

absolute increases in mean expenditures in column 4 are below the population's average for the first six deciles and above it for the remaing four deciles, pointing toward absolute Lorenz dominance by the 1980-81 expenditures distribution. ${ }^{4}$

An equivalent situation in income space is illustrated in Figure 1 in the twodimensional case. Line 1-1 represents all income distributions with the same mean income as distribution $\mathbf{x}$, while line 2-2 represents all income distributions with the same mean income as $\mathbf{y}$. Ray $\mathrm{O}-\mathrm{R}$ is the ray from the origin, which contains all income distributions with the same relative inequality as $\mathbf{x}$, while $\mathrm{A}-\mathrm{A}$ is the ray parallel to the $45^{\circ}$ line, which contains all income distributions with the same absolute inequality as $\mathbf{x}$. Present methods only allow us to say that distribution $\mathbf{y}$ falls somewhere on the line $2-2$ between rays $\mathrm{O}-\mathrm{R}$ and $\mathrm{A}-\mathrm{A}$. This paper claims that it is important to know whether the intermediate or centrist ray joining both distributions is nearer to $\mathrm{O}-\mathrm{R}$ or to $\mathrm{A}-\mathrm{A}$.

As will be seen below, our procedures allow the data to reveal a set of intermediate rays through $\mathbf{x}$ containing distribution $\mathbf{y}$. These rays serve to identify the set of centrist inequality notions for which $\mathbf{x}$ and $\mathbf{y}$ are equivalent in terms of inequality. For argument's sake, let us assume that distribution $\mathbf{y}$ can actually be placed in a single intermediate ray $\mathbf{I}-\mathrm{I}$ as in Figure 1. Then, for moderately minded people with a view of inequality between the ray I-I and the rightist or relative ray $\mathrm{O}-\mathrm{R}$, it could be said that social welfare would unambiguously increase by shifting from $\mathbf{x}$ to $\mathbf{y}$. Whereas for people of a more radical political tendency, whose concept of inequality lies between the ray I-I and the leftist or absolute ray $\mathrm{A}-\mathrm{A}, \mathbf{y}$ would show both a greater mean and a greater inequality than $\mathbf{x}$. In this case, both situations would be non-comparable in terms of social welfare.

${ }^{4}$ For a rigorous discussion about relative and absolute Lorenz dominance during the 1980s in Spain, see Del Río and Ruiz-Castillo (1996). For a complete summary of their results, see section III.2. 


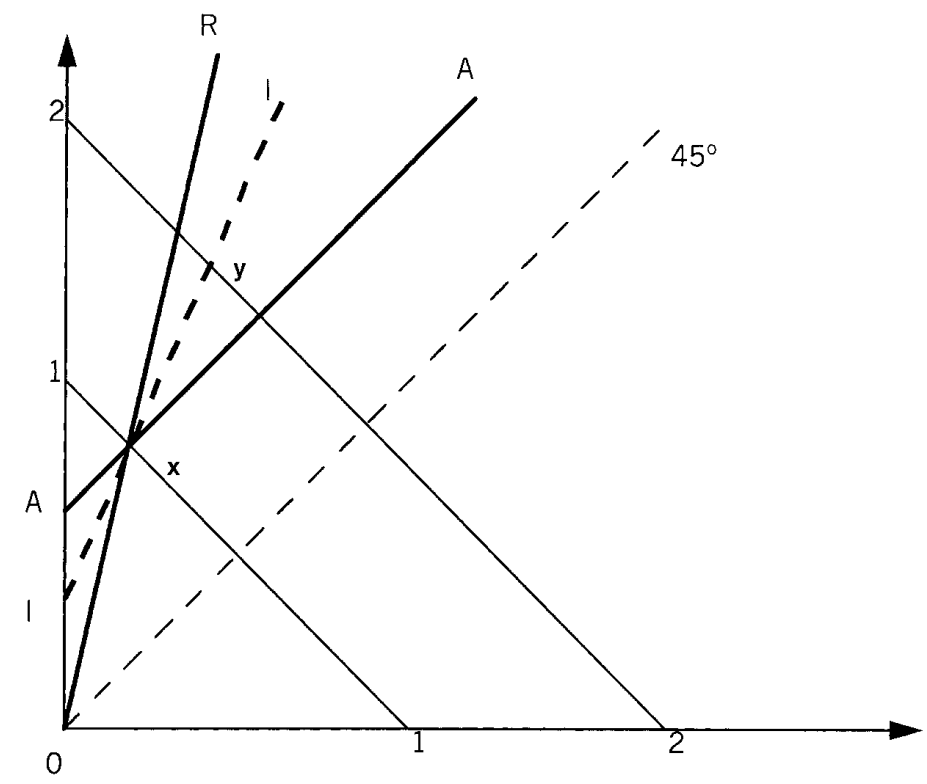

Figure 1. Constant (OR), Absolute (AA), and Intermediate (II) Income Inequality Viewed from Income Distribution $x$

An obvious alternative is to use specific inequality indices defined in the space of all possible income distributions. In this case, quantitative conclusions about the decrease in relative inequality or about the increase in the absolute one could be always obtained. In fact, using an appropriate SEF defined in that space, all doubts as to whether the increase in the mean compensates the increase in expenditures inequality would be also dispelled. However, we wish to offer the following three reasons for adopting the new approach:

(1) From the theoretical point of view, it seems interesting to use intermediate notions of inequality which respond to value judgments other than those normally held in the usual relative and absolute polar cases. Indeed, recent works based on inquiries into these issues have shown that people are by no means unanimous when choosing between a relative, an absolute or an intermediate inequality concept. ${ }^{5}$ Thus, the majority of scholars prefer a relative notion of inequality for technical or other reasons, while, because of the influence of their political attitudes toward redistribution or of other considerations of which we are unaware, most people appear to be in favor of an absolute or an intermediate concept of inequality. Then, as stated in the conclusions reached in Ballano and RuizCastillo (1993), perhaps the time has come to change the current consensus and

${ }^{5}$ See Amiel and Cowell (1999). In the Spanish case, Ballano and Ruiz-Castillo (1993) found that, for the subsample that showed an acceptable degree of consistency over the questionnaire, only 31 percent supported a relative view of inequality, 24 percent supported an absolute view, and 27 percent an intermediate notion (the rest supported other extreme views). 
use other types of inequality more often, as Kolm (1976a, 1976b) and Bossert and Pfingsten (1990), for example, have recommended.

(2) As far as the empirical work is concerned, it should be emphasized that the vast majority of the extensive literature available focuses on the measurement of relative inequality. There is also a handful of papers which measure absolute inequality - see, for instance, Blackorby, Donaldson, and Auersperg (1981) and Ruiz-Castillo (1998). However, even though there are various proposals for the measurement of intermediate inequality, there is still not a single empirical study on this topic.

(3) We have no doubts about the interest of using measurement instruments endowed with the property of completeness, but this strategy calls for additional strong assumptions which are unnecessary in this paper's approach. In general, it is advisable to evaluate the evolution of income inequality with the help of as few value judgments as possible. The reason is that the conclusions which may be reached with the minimalist approach that is proposed in this paper should be acceptable to a wider spectrum of people than those obtainable with complete inequality and social welfare indicators.

\section{A New Concept of Intermediate Inequality}

\section{II.1 Notation}

Let $\mathbf{x}=\left(x^{1}, \ldots, x^{H}\right) \in R_{++}^{H}, 2 \leq H<\infty$, denote an income or expenditures distribution with $x^{1} \leq \mathrm{x}^{2} \leq \ldots \leq x^{H}$. Then $D$ denotes the set of all possible ordered income distributions in $R_{++}^{H}$. Any real valued function $I$ defined on $D$ satisfying continuity, $S$-convexity and population replication invariance is called an income inequality measure. For later reference, let $S$ be the $H$-dimensional simplex and, for any $\mathbf{x} \in D$, let $\mathbf{v}_{x}=\left(v^{1}, \ldots, v^{H}\right) \in S$ be the vector of income shares with $v^{h}=x^{h} /$ $X$, where $X=\Sigma_{h} x^{h}$ is the aggregate income associated to distribution $\mathbf{x} .1$ denotes a row vector whose components are all ones, while e denotes the vector $(1 / H) \mathbf{1}$ in $S$.

\section{II.2 The $(\mathbf{x}, \pi)$-Inequality Concept}

An inequality index is said to be a relative inequality measure if it takes the same value along $H$-dimensional rays from the origin, i.e. whenever $I(\mathbf{x})=I(\lambda \mathbf{x})$ for all $\mathbf{x} \in D$ and for all $\lambda>0$ (scale invariance). An inequality index is said to be an absolute inequality measure if it adopts the same value along $H$-dimensional rays parallel to the line of equality, i.e., whenever $I(\mathbf{x})=I(\mathbf{x}+\mu \mathbf{1})$ for all $\mathbf{x} \in D$ and for all $\mu \in R$ such that $(\mathbf{x}+\mu \mathbf{1}) \in D$ (translation invariance).

Similarly, the simplest kind of intermediate inequality measures which is considered in this paper, requires that the set of income distributions with the same intermediate inequality is also a ray in $H$-dimensional space. An intermediate inequality concept of this kind is presented in DRRC, where those rays are constructed as follows. Given a reference distribution $\mathbf{x} \in D$ and a value of $\pi \in[0,1]$, consider the set $P_{(\mathbf{x}, \pi)}(\cdot)$ of all income distributions obtained in such a way that $\pi 100$ percent of any extra income is assigned to individuals according to the 
income share vector $\mathbf{v}$, and the remaining $(1-\pi) 100$ percent is allocated in equal absolute amounts. Formally:

$$
P_{(x, \pi)}(\mathbf{x})=\left\{\mathbf{y} \in D: \mathbf{y}=\mathbf{x}+\tau\left[\pi \mathbf{v}_{x}+(1-\pi) \mathbf{e}\right], \tau \in R\right\}
$$

A real function $I_{(x, \pi)}(\cdot): D \rightarrow R$ is said to be a $(\mathbf{x}, \pi)$-inequality measure if and only if $I_{(x, \pi)}(\mathbf{x})=I_{(x, \pi)}(\mathbf{y})$ for any $\mathbf{y} \in P_{(\mathbf{x}, \pi)}(\mathbf{x})$.

An example will help to understand this inequality notion. Let $\mathbf{x}=(200,800)$ and $\pi=0.5$. Then $\pi 100=50$ percent of all income differences are allocated according to the income shares vector $\mathbf{v}_{\mathbf{x}}=(1 / 5,4 / 5)$, and $(1-\pi) 100=50$ percent in equal absolute amounts according to the proportions $(1 / 2,1 / 2)$. Thus, the $(\mathbf{x}, \pi)$-ray of income distributions through $\mathbf{x}$ is given by

$$
P_{(\mathbf{x}, \pi)}(\mathbf{x})=\left\{\mathbf{y} \in R_{2 ;++}: \mathbf{y}=\mathbf{x}+\tau(7 / 2,13 / 20), \tau \in R\right\} .
$$

Suppose there are $\tau=100$ extra units of income. If thirty-five units are allocated to the first individual and sixty-five units to the second one according to the proportions $(7 / 20,13 / 20)$, then the new distribution $\mathbf{y}=(235,865)$ would have the same $(\mathbf{x}, \pi)$-inequality as $\mathbf{x}^{6}$

Informally, we may say that a value of $\pi=0.9$ reflects a center-right attitude, while a value of $\pi \quad 04$ reflects a center left perception of inequality The reason, of course, is that according to the first view, inequality is maintained if only 10 percent of any excess income is distributed according to the more demanding absolute criterion, while the second requires 60 percent to be allocated that way. On the other hand, notice that if $\pi=1,(\mathbf{x}, \pi)$-inequality becomes the relative view, whereas $\pi=0$ leads to the absolute view.

All intermediate inequality measures necessarily depend on an initial situation. Although some readers may find this a disadvantage, we agree with Pfingsten and Seidl (1997) when they assert that the meaning of "centrist" need not be decided universally, but can be made contingent on the situations we know and hence can evaluate well. In the previous numerical example, from the viewpoint of distribution $\mathbf{y}$ the two distributions would have the same $\left(\mathbf{y}, \pi^{\prime}\right)$ inequality, but $\pi^{\prime}$ would now be greater than $\pi=0.5$ - see Proposition 1 in DRRC. The interpretation is clear: the same centrist attitude is captured when, starting from $\mathbf{x}=(200,800), 50$ percent of the income difference between $X$ and $Y$ is allocated according to $\mathbf{v}_{\mathbf{x}}=(1 / 5,4 / 5)$, and 50 percent in equal absolute amounts, as when, starting from $\mathbf{y}, \boldsymbol{\pi}^{\prime}$ percent of the income difference is allocated according to $\mathbf{v}_{\mathbf{y}}=(0.21,0.79)$ and $\left(1-\pi^{\prime}\right)$ in equal absolute amounts. The reason why $\pi^{\prime}>0.5$ is that $\mathbf{y}$ has less relative inequality than $\mathbf{x}$. Thus, to get down to $\mathbf{x}$ from $\mathbf{y}$ so as to preserve intermediate inequality, the pattern $\mathbf{v}_{\mathbf{y}}$ can be more closely followed than the pattern $\mathbf{v}_{\mathbf{x}}$ from $\mathbf{x}$ - see DRRC for a more thorough discussion of this issue.

As shown in DRRC, our notion is a subset of the class of $\alpha$-invariant inequality measures discussed in Pfingsten and Seidl (1997). The reason for choosing the $(x, \pi)$-inequality concept is twofold. In the first place, it has a clear economic interpretation, which is not the case of the $\alpha$-invariant concept. In the

\footnotetext{
${ }^{6}$ Technically speaking, the definition of $(\mathbf{x}, \pi)$-inequality does not call for the distribution whose inequality we want to evaluate to coincide with the reference distribution $\mathbf{x}$. For the properties and further details of this notion, see DRRC.
} 
second place, it can be made operational by means of certain comparisons according to the usual Lorenz criterion. ${ }^{7}$

\section{II.3 Operational Methods: The Homogeneous Case}

For any pair of income distributions $\mathbf{x}, \mathbf{y} \in D$, let $\mathbf{v} x L \mathbf{v}_{\mathbf{y}}$ denote weak Lorenz dominance. Let also $m(\mathbf{x})$ represent the mean of distribution $\mathbf{x}$. For any $\mathbf{x} \in D$ and $\pi \in[0,1]$, denote by $W_{(\mathbf{x}, \pi)}$ the class of SEFs which, apart from the three common assumptions of continuity, $S$-concavity and population replication invariance, have a social preference for efficiency consistent with $(\mathbf{x}, \pi)$-inequality. Let us identify $\mathbf{x}$ and $\mathbf{y}$ with the 1980-81 and 1990-91 Spanish income distributions at constant prices, respectively. Then we know that $m(\mathbf{y})>m(\mathbf{x}), \mathbf{v}_{y} \operatorname{L} \mathbf{v}_{x}$ but $\mathbf{e} L \mathbf{v}_{y}$. The question we want to answer is the following: for which $\pi$ values can we conclude that $W_{(\mathbf{x}, \pi)}(\mathbf{y})>W_{(\mathbf{x} \pi)}(\mathbf{x})$ ?

There is no a priori given reasonable set of $\pi$ values to take into account in this social welfare evaluation exercise. The paper's strategy is to allow the data to provide an answer to the above question. One proceeds as follows. Fix $\tau=Y_{-}$ $X>0$, and define the set of rays

$$
\mathbf{c}(\pi) \quad \mathbf{x}+\tau\left[\pi \mathbf{v}_{x}+\left(\begin{array}{ll}
1 & \pi
\end{array}\right) \mathbf{e}\right], \pi \in[0,1]
$$

Notice that for any $\pi, \mathbf{c}(\pi)$ has the same mean as $\mathbf{y}$ and the same $(\mathbf{x}, \pi)$-inequality as $\mathbf{x}$. If there were to exist a $\pi^{*}$ in the unit interval such that $\mathbf{y}=\mathbf{c}\left(\pi^{*}\right)$ then we would be done. The reason is that, for any $\pi>\pi^{*}, \mathbf{y}$ would have a smaller $(\mathbf{x}, \pi)$ inequality than $\mathbf{x}$. Taking into account that $m(\mathbf{y})>m(\mathbf{x})$, we would conclude that $W(\mathbf{y})>W(\mathbf{x})$ for all SEFs $W(\cdot)$ belonging to the class $W_{(\mathbf{x}, \pi)}(\cdot)$ for all $\pi \in\left[\pi^{*}, 1\right]$. In general, there will be no such $\pi^{*}$. In other words, the intermediate space to which $\mathbf{y}$ belongs, that is, the set of all income distributions with less relative inequality but more absolute inequality than $\mathbf{x}$, is far richer than the set of distributions attainable by choosing successive values of $\pi$ in equation (1) - for the relationship of these two sets in a three-dimensional example, see DRRC.

What we can be certain of, is that there is a range of $\pi$ values for which $\mathbf{y}$ has more $(\mathbf{x}, \pi)$-inequality than $\mathbf{x}$, another set for which it has less, and another set for which both distributions are non-comparable from the point of view of $(\mathbf{x}, \pi)$-inequality. Thus, what is done in the general case is to find a partition of the unit interval into these three subsets. In the first place, by making the pertinent Lorenz curves comparisons, one searches empirically for the lower value of $\pi, \pi_{2}$, such that $\mathbf{y} L \mathbf{c}(\pi)$ for any $\pi \in\left[\pi_{2}, 1\right]$. Then, by applying Theorem 1 in DRRC, it can be concluded that $W(\mathbf{y})>W(\mathbf{x})$ for all the SEFs $W(\cdot)$ belonging to the class $W_{(\mathbf{x}, \pi)}(\cdot)$ for all $\pi \in\left[\pi_{2}, 1\right]$. In the second place, one searches for the greater value of $\pi, \pi_{1}$, such that $\mathbf{c}(\pi) L \mathbf{y}$ for any $\pi \in\left[0, \pi_{1}\right]$. For the centrist inequality views represented by that range of $\pi$ values, $I_{(\mathbf{x}, \pi)}(\mathbf{y})>I_{(\mathbf{x}, \pi)}(\mathbf{x})$. Since $m(\mathbf{y})>m(\mathbf{x})$, for this range of $\pi$ values an unambigous conclusion in terms of welfare cannot be

\footnotetext{
${ }^{7}$ There are other ray-invariant intermediate inequality notions, like the one suggested by Kolm (1976a, 1976b) or the single parameter $\mu$-inequality concept proposed by Bossert and Pfingsten (1990). Unfortunately, as pointed out by Pfingsten and Seidl (1997), the latter has a serious disadvantage: it approaches the rightist position when aggregate income rises, even if the income distribution becomes more unequal according to an inequality measure. For another concept in which the set of income distributions with the same intermediate inequality is a parabola, see Krtscha (1994).
} 
obtained. Finally, since for any $\pi \in\left(\pi_{1}, \pi_{2}\right)$ the two distributions are non-comparable in terms of $(\mathbf{x}, \pi)$-inequality, in this third case no conclusion in terms of welfare can be reached either.

\section{II.4. Operational Methods: The Heterogeneous Case}

Let us now admit that we have a population of $h=1, \ldots, H$ households which can differ in income, $x^{h}$, and/or a vector of household characteristics. In this paper, it is assumed that households of the same size have the same needs and, therefore, their incomes are directly comparable. (See the Appendix where each of the subgroups in the basic partition by household size is investigated.) However, social evaluation within subgroups need not yield unanimous results. Moreover, it is always convenient to extract conclusions for the population as a whole. Therefore, a procedure is needed to establish inter-household welfare comparisons. This is, of course, the role played by equivalence scales.

Assume that there are $k=1, \ldots, K$ household sizes. Denote by $z^{h}$ the equivalent or the adjusted income of household $h$. Let $\mathbf{x}^{k}$ and $\mathbf{z}^{k}$ be the vectors of original incomes and adjusted incomes, respectively, for households of size $k$. In principle, we find that it is reasonable to impose the following two conditions on any equivalence scales procedure.

Condition 1. For any household $h$ of size $k, z^{h}=F\left(x^{h}, k, \Theta\right)$, where $\Theta \in R$.

Condition 2. For any $k$ and any $\pi \in[0,1], I_{\left(\mathbf{x}^{k}, \pi\right)}\left(\mathbf{z}^{k}\right)=I_{\left(\mathbf{x}^{k}, \pi\right)}\left(\mathbf{x}^{k}\right)$.

Condition 1 reflects a normative value judgment according to which household $h$ 's adjusted income, being an individual concept, should be independent from other individuals' characteristics. In other words, it should only depend on individual features - that is, the unadjusted income $x^{h}$, and the household size $k$-as well as on a single dimensional parameter $\Theta$ whose role would be to characterize the economies of scale in consumption within the household. Condition 2 requires that, whatever the inequality concept we care to use, within each ethically homogeneous subgroup the adjustment process should not alter the underlying inequality: the $\left(\mathbf{x}^{k}, \pi\right)$-inequality of adjusted income should be equal to the $\left(\mathbf{x}^{k}, \pi\right)$ inequality of original income.

Condition 2 implies that for any $h$ of size $k$,

$$
z^{h}=x^{h}-\tau^{k}\left[\pi\left(x^{h} / m\left(\mathbf{x}^{k}\right)+(1-\pi) \mathbf{1}\right] \text {, for some } \tau^{k} \in R .\right.
$$

In this way, household $h$ 's adjusted income $z^{h}$ belongs to ray $P_{\left(\mathbf{x}^{k}, \pi\right)}\left(\mathbf{x}^{k}\right)$, so that the $\left(\mathbf{x}^{k}, \pi\right)$-inequality remains constant. In the relative case, i.e. when $\pi=1$, we have:

$$
z^{h}=x^{h}\left[1-\left(\tau^{k} / m\left(\mathbf{x}^{k}\right)\right)\right] .
$$

In order for condition 1 to be satisfied, we must have $\tau^{k}=f(k, \Theta) m\left(\mathbf{x}^{k}\right)$. In particular, Buhmann et al. (1988) and Coulter et al. (1992a, 1992b) suggest the following specification: $f(k, \Theta)=\left(k^{\Theta}-1\right) / k^{\Theta}, \Theta \in[0,1]$. Then, adjusted income for any household $h$ becomes:

$$
z^{h}(k, \Theta)=x^{h} / k^{\Theta}, \Theta \in[0,1] .
$$


This procedure recognizes that larger households have greater needs, but also greater opportunities to achieve economies of scale in consumption. Taking a single adult as the reference type, the expression $k^{\Theta}$ can be interpreted as the number of equivalent adults in a household of size $k$. Thus, the greater $\Theta$ is, the greater the number of equivalent adults for each household or, in other words, the smaller the economies of scale. When $\Theta=0$ and economies of scale are assumed to be infinite, adjusted income coincides with unadjusted household income; while if $\Theta=1$ and economies of scale are completely ruled out, then adjusted income equals per capita household income. Notice that, given $\Theta$, the number of equivalent adults is a non-linear increasing function of $k$.

Next, consider the absolute case in which $\pi=0$. Equation (2) becomes:

$$
z^{h}=x^{h}-\tau^{k} \in R .
$$

Consider the specification $\tau^{k}=\Theta(k-1)$ which satisfies condition 1. In this case, adjusted income becomes:

$$
z^{h}=x^{h}-\Theta(k-1) .
$$

The parameter $\Theta$ can be interpreted now as the cost of an equivalent adult. The problem is that such a cost is independent of household size. In search of a better empirical specification, Ruiz-Castillo (1998) requires that the mean of the adjusted income distribution in the absolute case should be the same as the one obtained in the relative case. That is, the following additional condition is imposed:

Condition 3. For each $k$, let $\mathbf{z}^{k}(k, \Theta)$ denote the vector of adjusted incomes of households of size $k$ in the relative case. Then, for any other equivalence scales procedure which gives rise to a vector $\mathbf{z}^{k}$ of adjusted incomes, we must have: $m\left(\mathbf{z}^{k}\right)=m\left(\mathbf{z}^{k}(k, \Theta)\right)$.

In the absolute case this condition implies that $\tau^{k}=m\left(\mathbf{x}^{k}\right)-m\left(\mathbf{z}^{k}(k, \Theta)\right)$. If the widely applied specification presented in equation (3) for the relative case is accepted, then adjusted income is equal to:

$$
z^{h}=x^{h}-m\left(\mathbf{z}^{k}(k, \Theta)\right)\left(k^{\Theta}-1\right) .
$$

Compare equations (4) and (5). On one hand, the cost of an adult is now equal to the mean income per equivalent adult. Moreover, rather than the number of persons less one, such a cost is now multiplied by the number of equivalent adults less one. Thus, as in the relative case, the greater $\Theta$ is for any $k$, the smaller the economies of scale are within the household. However, on the other hand, condition 1 has to be sacrificed.

Finally, consider the intermediate case in which $\pi \in(0,1)$. Let us make $\tau^{k}=$ $g\left(k, \Theta, m\left(\mathbf{x}^{k}\right)\right)$ in order to rewrite equation (2) as follows:

$$
z^{h}=x^{h}-g\left(k, \Theta, m\left(\mathbf{x}^{k}\right)\right)\left[\pi\left(x^{h} / m\left(\mathbf{x}^{k}\right)+(1-\pi) \mathbf{1}\right]=x^{h}-s\left[k, \Theta, m\left(\mathbf{x}^{k}\right)\right] .\right.
$$

Clearly, the function $s\left[k, \Theta, m\left(\mathbf{x}^{k}\right)\right]$ cannot be made independent from $m\left(\mathbf{x}^{k}\right)$ for any value of $\pi \in(0,1)$. That is to say, in the intermediate case conditions 1 and 2 are incompatible. The question is: how should we proceed in practice? There are two alternatives. Either the adjustment procedure of equation (3) for the relative case is accepted, sacrificing condition 2. Or the dependency of function 
$s\left[k, 1, m\left(\mathbf{x}^{k}\right)\right]$ on $m\left(\mathbf{x}^{k}\right)$ is accepted, sacrificing condition 1 . In this case, given what has been learned from the absolute case, condition 3 is required to be satisfied. Given also the specification of equation (3), it is easy to see that

$$
\tau^{k}=g\left[k, \Theta, m\left(\mathbf{x}^{k}\right)\right]=m\left(\mathbf{z}^{k}(k, \Theta)\right)\left(k^{\Theta}-1\right) .
$$

Thus, again, the greater $\Theta$ is for any $k$, the greater is $\tau^{k}$ and the smaller the economies of scale are within the household. This second alternative which satisfies conditions 2 and 3 is the one which is chosen in this paper.

\section{EMPIRICAL RESULTS}

\section{III.1. The Data}

As indicated in the Introduction, our data come from the 1980-81 and 199091 EPFs - available at http://www.eco.uc3m.es/epf.html. The EPFs are large, comparable household budget surveys of 23,972 and 21,155 observations, respectively, for a population of approximately 10 or 11 million households, or 37 and 38 million persons, occupying residential housing in all of Spain including the North African cities of Ceuta and Melilla.

Household welfare is approximated by a measure of current consumption, namely, household total current expenditure on private goods and services, net of expenditures on the acquisition of certain durables, but inclusive of imputations for self-consumption, wages in kind, meals subsidized at work, and the rental value for owner-occupied and other non-rental housing. ${ }^{8}$ Total household expenditures are expressed at constant prices of the winter of 1991 by means of household specific statistical price indices. ${ }^{9}$ Since the interest is in personal rather than household welfare, we follow the usual practice of studying the personal distribution in which each person is assigned the adjusted expenditures of the household to which he or she belongs. All estimates use the blowing up factors provided by the INE to obtain population rather than sample estimates.

Table 2 presents the change in mean household expenditures and demographic information for both the partition by household size and the population as a whole. In the latter case, the results are presented as a function of $\Theta$, the parameter which reflects different alternatives about the generosity of the equivalence scales. Smaller households consisting of one to four persons are more important at the end of the decade, and the opposite is the case for larger households. Thus, whereas the household population grows by more than 10 percent, the number of persons only increases by approximately 4 percent. Correspondingly, household size decreases from 3.7 in $1980-81$ to 3.41 in 1990-91.

There is an important growth of mean household expenditures in real terms over the decade for all household types. Single person households and the large group of four-person households, experience an increase in the mean of more than 30 percent. At the opposite end, large households of seven persons grow only about 17 percent. The increase for all other households falls, approximately,

\footnotetext{
${ }^{8}$ See Ruiz-Castillo (1998) for a discussion justifying why with EPF data this measure is the best proxy for a household standard of living.

${ }^{9}$ For an explanation of the construction of these indexes, see Ruiz-Castillo et al. (2000).
} 
TABLE 2

Percentage Distribution by Persons in the Partition by Household Size and Mean Household Expenditures at Winter 1991 Prices: 1980-81 versus 1990-91

\begin{tabular}{lcccccccc}
\hline \hline & \multicolumn{7}{c}{ Household Size } \\
\cline { 2 - 9 } Personal Distr. in \% & 1 & 2 & 3 & 4 & 5 & 6 & 7 & All \\
\hline $1980-81$ & 2.1 & 11.4 & 15.1 & 25.5 & 20.1 & 12.5 & 6.8 & 93.5 \\
$1990-91$ & 2.9 & 13.1 & 18.3 & 29.3 & 19.4 & 9.6 & 4.4 & 97.0 \\
\hline
\end{tabular}

Percentage Change from 1980-81 to 1990-91 in Mean Household Expenditures, in \%

\begin{tabular}{lccccccc} 
Household Size & 1 & 2 & 3 & 4 & 5 & 6 & 7 \\
\hline & 35.5 & 24.7 & 26.7 & 31.3 & 27.4 & 28.0 & 16.9 \\
Population as a whole & & & $\Theta=0.0$ & $\Theta=0.4$ & $\Theta=0.7$ & $\Theta=1.0$ \\
& & 22.5 & 26.8 & 30.1 & 33.2 \\
\hline
\end{tabular}

in the $25 / 28$ percent range. For the population as a whole, the smaller the economies of scale, the greater the growth in mean adjusted expenditure, which varies between 22.5 and 33.2 percentage points.

\section{III.2. Previous Results}

According to Shorrocks (1983) and Moyes (1987), one income distribution provides greater social welfare than another according to all the SEFs in class $W_{R}$ (or $W_{A}$ ), if and only if the first one has a larger mean and dominates the second distribution in the relative (or absolute) Lorenz sense. To test whether this is the case, Del Río and Ruiz-Castillo (1996) use asymptotically distribution-free inference procedures developed by Bishop et al. $(1989,1994) .{ }^{10}$ The main findings are the following:

(1) For one-, two-, three- and five-member households, the 1990-91 household expenditures distribution dominates the 1980-81 one according to the relative Lorenz criterion. However, for four-, six- and seven-member households, both distributions are statistically equivalent in the relative Lorenz sense.

(2) The 1990-91 household expenditures distribution for the total population Lorenz-dominates the $1980-81$ one for all $\Theta$ values. Taking into account that the 1990-91 mean household expenditures is always significantly greater than the 1980-81 one, it can be concluded that in all cases there has been an unambiguous increase in relative welfare according to all the SEFs in the class $W_{R}$.

(3) The large increases in the mean, which cause absolute inequality to increase ceteris paribus, outweigh the decrease in relative inequality in all of the cases just reported. Thus, during the 1980s there has been a generalized increase in absolute household expenditures inequality for all household sizes as well as

\footnotetext{
${ }^{10}$ Unlike the classical tests — see Beach and Davidson (1983), for instance — which only provide a partition of the sample space into two regions - acceptance and rejection regions - the procedure used by Bishop et al. $(1989,1994)$, based in the union-intersection principle makes it possible to distinguish between three differentiated regions associated with dominance, equality and non-comparability between the two situations under comparison. Richmond (1982) presents the methodology used to construct joint confidence intervals. Beach and Kaliski (1986) have extended this methodology to samples which, like ours, involve weighted observations.
} 
for the total population. Therefore, no unambiguous conclusions can be obtained in terms of all the SEFs in the class $W_{A}$.

\section{III.3 Results on Intermediate Inequality for the Population as a Whole}

The results just summarized constitute a textbook example for an application of a centrist approach. Let us denote by $\mathbf{x}$ and $\mathbf{y}$ the 1980-81 and 1990-91 distributions, respectively. In terms of the notation introduced in section II, we must search for a pair of values $0 \leq \pi_{1} \leq \pi_{2} \leq 1$, where at least the first or the last inequality is strict. As can be seen in the Appendix, there are important differences in the social evaluation of households of different sizes. How do these differences become aggregated at the population level? In principle, the answer depends on the way household size is taken into account in the definition of adjusted household expenditure. In the Spanish case, an important finding is that the results in Table 3 for the total population are rather robust to the choice of the equivalence scales parameter $\Theta$.

TABLE 3

A Comparison of THE 1980-81 versus 1990-91 HousEHOLd EXPENDITURES Distributions FROM AN INTERMEDIATE INEQUALity Point of View: $\pi$-VAlues For the Population as a Whole as a Function of the Equivalence Scales Parameter $\Theta$

\begin{tabular}{ccc}
\hline \hline$\Theta$ & $\pi_{2}$ & $\pi_{1}$ \\
\hline 0.1 & 0.89 & 0.75 \\
0.4 & 0.87 & 0.71 \\
0.7 & 0.86 & 0.73 \\
1.0 & 0.88 & 0.75 \\
\hline
\end{tabular}

Basically, for a relatively small set of centrist attitudes according to which inequality is maintained if 11-14 percent or less of any excess aggregate expenditures is distributed in absolute amounts, there is a decrease in inequality. For all those who think that inequality is maintained if at least $25-29$ percent is distributed in absolute amounts, inequality has increased. For the rest, inequality differences are not statistically significant. Taking into account the increase in the mean household expenditures, social welfare has unambiguously increased for all SEFs in the class $W_{(\mathbf{x}, \pi)}$, where $\pi[0.75,1]$.

\section{Concluding Remarks}

Using empirical methods developed by Shorrocks (1983) and Moyes (1987), it can be determined whether an income distribution $\mathbf{y}$ has a greater mean income, less relative inequality, but more absolute inequality than distribution $\mathbf{x}$. This is exactly the case when $\mathbf{x}$ and $\mathbf{y}$ are taken to be, respectively, the 1980-81 and 199091 household expenditures distributions, adjusted for household size, following a decade of Socialist governments in Spain. What present methods cannot say is 
whether the reduction in relative inequality (or the increase in absolute inequality) during the 1980s was "large" or "small."

This is a situation where centrist or inequality measures of inequality are called for. This paper has presented the first empirical application of this kind of inequality concept. Among the ray-invariant intermediate inequality measures, the $(\mathbf{x}, \pi)$-inequality notion introduced in DRRC is used because it has a clear economic interpretation and it can be made operational in the Shorrocks way. To deal with the heterogeneous case, total expenditures of households of the same size are assumed to be directly comparable, and an axiomatic justification for a widely used single parameter model of equivalence scales is provided.

Which are the politically correct centrist attitudes toward inequality to be used in a given case? Lacking an answer to this question, our empirical procedure allows the data to reveal for what type of intermediate inequality notions the situation in 1990-91 in Spain is statistically equivalent or non-comparable to the situation in 1980-81. This also reveals for what type of centrist attitudes there has been a reduction or an increase in inequality during that period.

The results can be summarized as follows. In the first place, for small households of three or fewer persons, inequality has decreased for those views according to which inequality is maintained if 20 percent or less of any excess aggregate expenditures is distributed in absolute amounts - while the rest is distributed according to the household expenditures shares in the 1980-81 distribution. For larger households, intermediate inequality has either remained constant or has increased. In the second place, for the population as a whole the results are rather robust to the importance we give to the economies of scale within the household: inequality has decreased for those views according to which inequality is maintained if 11-14 percent or less of any excess aggregate expenditures is distributed in absolute amounts. Globally, we take these results as implying that the decrease in intermediate inequality in Spain during this period has been "small."

Nevertheless, in the international context among OECD countries this performance is rather remarkable. As analyzed in detail in Del Río and Ruiz-Castillo (2001), the extension of the coverage of the Social Security and the unemployment subsidy systems, the increase in real terms of the minimum pension, the decrease in the agricultural population coupled with a generous policy of agricultural subsidies, among other factors, are all significant forces which help to account for the reduction of inequality during this period. From this perspective, the Spanish experience could be of some interest to other countries undergoing a political and economic transition, both in Latin America and in Eastern Europe.

A final remark is in order. Let us assume that the 1990 income distribution has both a greater mean and more relative inequality according to the usual Lorenz criterion than the 1980 income distribution. Assume, however, that the 1990 distribution dominates the 1980 one according to the generalized Lorenz criterion. This is the situation in the UK, for example. In terms of Figure 1, the present methods allow us to conclude that the 1990 UK distribution falls in the line $2-2$ to the left of the relative ray $\mathrm{O}-\mathrm{R}$, and to the right of a ray drawn through $\mathbf{x}$ but parallel to the vertical axis, which we will call a Paretian ray. The question is: how can it be known-without using specific inequality measures which require additional value judgments - whether the 1990 distribution is 
"nearer" the ray $\mathrm{O}-\mathrm{R}$, reflecting a small increase in relative inequality, or "nearer" the Paretian ray, reflecting the opposite situation? Del Río (1996) extends the methods presented in this paper to provide an operative answer to this question.

\section{Appendix: Results in the Partition by Household Size}

As in the case of the entire population, in each subgroup of households of the same size a pair of values $\pi_{1}$ and $\pi_{2}$ must be found, with $0 \leq \pi_{1} \leq \pi_{2} \leq 1$, where at least the first or the last inequality is strict. The results are presented in Table A-1. Household sizes are ordered, first, by the minimum $\pi_{2}$ value, then by the maximum $\pi_{1}$ value.

TABLE A-1

A COMPARISON OF THE 1980-81 versus 1990-91 HousEHOLd EXPENDITURES

DisTRIBUTIONS FROM AN INTERMEDIATE

INEQuality Point of View: $\pi$-VAlues

Within the Partition by Household Size as a Function of THE Equivalence Scales Parameter $\Theta$

\begin{tabular}{lcc}
\hline \hline Household Size & $\pi_{2}$ & $\pi_{1}$ \\
\hline 3 persons & 0.79 & 0.49 \\
1 person & 0.80 & 0.71 \\
2 persons & 0.80 & 0.60 \\
5 persons & 0.96 & 0.61 \\
4 persons & 1.00 & 0.83 \\
6 persons & 1.00 & 0.65 \\
7 persons & 1.00 & 0.07 \\
\hline
\end{tabular}

Let us begin with three-person households for whom $\pi_{2}=0.79$ and $\pi_{1}=0.49$. Consider the class of center-right attitudes for which inequality is maintained as long as $\left(1-\pi_{2}\right) 100=(1-0.79) 100=21$ percent or less of any excess income is distributed in absolute amounts, while the remaining amount is distributed according to the 1980-81 household expenditures shares. For this class inequality has decreased during the 1980s. For all center-left people for whom inequality is maintained only if at least $\left(1-\pi_{1}\right) 100=(1-0.49) 100=51$ percent of any excess income is distributed in absolute amounts, inequality has increased. For those in between, both distributions are statistically equivalent. Taking into account that mean household expenditures have increased by 26.7 percent, for three-person households social welfare has increased unambiguously for all SEFs in the class $W_{(\mathbf{x}, \pi)}$, where $\pi \in[0.49,1]$. There is nothing that can be said about social welfare for people whose intermediate notion of inequality is represented by a lower $\pi$ value.

A similar analysis can be made for one-, two- and five-person households. The situation for all other household sizes for which $\pi_{2}=1.0$ is quite different. Let us take four-person households, for instance. As far as inequality is concerned, the only statement that can be supported is that for the class of centerright attitudes for which inequality is maintained if 17 percent or less of any excess 
income is distributed in absolute amounts, inequality is equivalent in both situations. Therefore, for those intermediate inequality views, social welfare has increased. For the rest of the people with a centrist perception of inequality, the four-person household expenditures inequality has increased during this period. Hence, no conclusion can be obtained in terms of the class of SEFs $W_{(\mathbf{x}, \pi)}$ for all $\pi \in[0,0.83]$.

\section{REFERENCES}

Amiel, Y. and F. A. Cowell, Thinking About Inequality, Cambridge University Press, Cambridge, 1999.

Atkinson, A. B., L. Rainwater, and T. Smeeding, Income Distribution in OECD Countries: the Evidence from the Luxembourg Income Study (LIS), OECD, Paris, 1995.

Ballano, C. and J. Ruiz-Castillo, Searching by Questionnaire for the Meaning of Income Inequality, Revista Española de Economía, 10, 233-59, 1993.

Beach, C. M. and R. Davidson, Distribution-free Statistical Inference with Lorenz Curves and Income Shares, Review of Economic Studies, 50, 723-35, 1983.

Beach, C. M. and S. Kaliski, Lorenz Curve Inference with Sample Weights: An Application to the Distribution of Unemployment Experience, Applied Statistics, 35, 28-45, 1986.

Bishop, J., J. Formby, and P. Thistle, Statistical Inference, Income Distributions, and Social Welfare, in D. J. Slotje (ed.), Research on Economic Inequality, Vol. I, Jay Press, Greenwich, CT, 1989.

Bishop, J., S. Chakravorti, and P. Thistle, Relative Inequality, Absolute Inequality, and Welfare: Large Sample Tests for Partial Orders, Bulletin of Economic Research, 46, 41-59, 1994.

Blackorby, C., D. Donaldson, and M. Auersperg, A New Procedure for the Measurement of Inequality Within and Among Population Subgroups, Canadian Journal of Economics, 14, 665$85,1981$.

Bossert, W. and A. Pfingsten, Intermediate Inequality: Concepts, Indices, and Welfare Implications, Mathematical Social Sciences, 19, 117-34, 1990.

Buhmann, B., L. Rainwater, G. Schmaus, and T. Smeeding, Equivalence Scales, Well-Being, Inequality and Poverty: Sensitivity Estimates Across Ten Countries Using the Luxembourg Income Study Database, Review of Income and Wealth, 34, 115-42, 1988.

Coulter, F., F. Cowell, and S. Jenkins, Differences in Needs and Assessment of Income Distributions, Bulletin of Economic Research, 44, 77-124, 1992a.

- Equivalence Scale Relativities and the Extent of Inequality and Poverty, Economic Journal, 102, 1067-82, $1992 b$.

Del Río, C., Desigualdad y pobreza en España, de 1980-81 a 1990-91, unpublished PhD dissertation, Universidad Carlos III, 1996.

Del Río, C. and J. Ruiz-Castillo, Ordenaciones de bienestar e inferencia estadística. El caso de las EPF de 1980-81 y 1990-91, in La desigualdad de recursos. Segundo Simposio sobre la distribución de la renta y la riqueza, Fundación Argentaria, Colección Igualdad, Volumen VI, 9-44, 1996. -, Intermediate Inequality and Welfare, Social Choice and Welfare, 17, 223-39, 2000.

- Accounting for the Decline in Spanish Household Expenditures Inequality During the 1980s, forthcoming in Spanish Economic Review, 2001.

Gottschalk, P. and T. Smeeding, Crossnational Comparisons of Earnings and Income Inequality, Journal of Economic Literature, 35, 633-86, 1997.

Gouveia, M. and J. Tavares, The Distribution of Household Income and Expenditure in Portugal: 1980 and 1990, Review of Income and Wealth, 41(1), 1-17, 1995.

Jimeno, J. F., O. Cantó, A. Cardoso, M. Izquierdo, and C. Rodrigues, Integration and Inequality: Lessons from the Accessions of Portugal and Spain to the EU, mimeo, 2000.

Kolm, S. C., Unequal Inequalities I, Journal of Economic Theory, 12, 416-42, 1976a. , Unequal Inequalities II, Journal of Economic Theory, 13, 82-111, $1976 \mathrm{~b}$.

Krtscha, M., A New Compromise Measure of Inequality, in W. Eichorn (ed.), Models and Measurement of Welfare and Inequality, 1994.

Lambert, P., The Distribution and Redistribution of Income: A Mathematical Analysis, Manchester University Press, Manchester, 1993.

Martin C., Spain in the New Europe, Macmillan, London, 1999.

Moyes, P., A New Concept of Lorenz Domination, Economics Letters, 23, 203-7, 1987.

Pfingsten, A. and C. Seidl, Ray Invariant Inequality Measures, in S. Zandvakili and D. Slotje (eds), Research on Taxation and Inequality, 107-29, Jay Press, Greenwich, CT, 1997. 
Richmond, J., A General Method for Constructing Simultaneous Confidence Intervals, Journal of the American Statistical Association, 77, 455-60, 1982.

Rodrigues, C. F., Measurement and Decomposition of Inequality in Portugal, 1980/81-1990/91, Department of Applied Economics, University of Cambridge, ESRC Discussion Paper, MU 9302, 1993.

Ruiz-Castillo, J., A Simplified Model for Social Welfare Analysis. An Application to Spain, 1973-74 to 1980-81, Review of Income and Wealth, 44(1), 123-41, 1998.

Ruiz-Castillo, J., C. Higuera, M. Izquierdo, and M. Sastre, Series de precios individuales para las EPF de 1973-74, 1980-81 y 1990-91 con base en 1976, 1983 y 1992, mimeo, available at http:// www.eco.uc3m.es/investigacion/epf.html, 2000.

Shorrocks, A., Ranking Income Distributions, Economica, 50, 3-17, 1983. 